\title{
Blow-up solutions to the Cauchy problem of a fractional reaction-diffusion system
}

\section{Ezi Wu and Yanbin Tang*}

\section{${ }^{*}$ Correspondence:}

tangybhust@sina.com

School of Mathematics and

Statistics, Huazhong University of

Science and Technology, Wuhan,

Hubei 430074, P.R. China

\begin{abstract}
In this paper, we study the blow-up property of positive mild solutions to the Cauchy problem of a system of fractional reaction-diffusion equations. For the fundamental solution $P(t, x)$ of the fractional heat operator $\partial_{t}+(-\triangle)^{\frac{\beta}{2}}$ defined on the whole space $R^{N}$, due to the properties of $P(t, x)$ established by $\mathrm{H}$ Yosida and some estimates of $P(t, x)$ developed by $L$ Caffarelli and A Figalli, we first use an iteration method to establish the estimates of lower bounds of positive mild solutions; then we obtain the unboundedness of solutions for large time. Finally we give a sufficient condition that the positive mild solution to a fractional reaction-diffusion system blows up in finite time.
\end{abstract}

MSC: $35 K 20 ; 35 K 55$

Keywords: fractional Laplacian; fractional reaction-diffusion system; blow-up

\section{Introduction}

In this paper, we consider the blow-up solutions to the Cauchy problem of a system of fractional reaction-diffusion equations,

$$
\left\{\begin{array}{l}
u_{t}+(-\Delta)^{\frac{\beta}{2}} u=v^{p}, \quad x \in R^{N}, t>0, \\
v_{t}+(-\Delta)^{\frac{\beta}{2}} v=u^{q}, \quad x \in R^{N}, t>0, \\
u(0, x)=u_{0}(x) \geq 0, \quad v(0, x)=v_{0}(x) \geq 0, \quad x \in R^{N},
\end{array}\right.
$$

where $(-\Delta)^{\frac{\beta}{2}}$ for $0<\beta \leq 2$ is called the fractional power of the Laplacian operator $-\Delta$, and $u_{0}(x), v_{0}(x)$ are nonnegative continuous functions defined on $R^{N}(N \geq 1), p, q$ are positive constants.

Systems such as (1.1) arise in the fields like molecular biology, hydrodynamics, and statistical physics [1].

We can find the definition and some elementary properties of the fractional Laplacian operator in Yosida [2]. The applications of fractional Laplacian operators can be found in different fields, we refer the readers to Caffarelli et al. [3-5], Wang and Tang [6, 7], and so on.

It is well known that, for an initial value problem of a pure reaction equation with a positive source term,

$$
\frac{d u}{d t}=f(u), \quad u(0)=u_{0},
$$


its solution blows up in finite time if and only if

$$
T\left(u_{0}\right)=\int_{u_{0}}^{+\infty} \frac{1}{f(s)} d s<+\infty .
$$

The diffusion process is one of the important processes in mathematical theories and real world problems [8-12]. The diffusion and/or the boundary conditions have the tendency to decrease or increase the solution [13]. This means that, for some reactiondiffusion equations, the blow-up of solutions may occur, though the corresponding ordinary differential equation (ODE) possesses a global attractor. This is called diffusioninduced blow-up.

Kaplan studied for the first time in a seminal paper [14] the blow-up phenomenon of reaction-diffusion equations in bounded domains. He showed that for a convex source term $f(u)$ satisfying (1.3) diffusion cannot prevent blow-up if the initial state is large enough. Escobedo and Herrero $[15,16]$ described the global existence and blow-up of the solutions of the system (1.1) with $\beta=2$ on a bounded domain, that is,

$$
\left\{\begin{array}{l}
u_{t}-\Delta u=v^{p}, \quad x \in \Omega, t>0, \\
v_{t}-\Delta v=u^{q}, \quad x \in \Omega, t>0, \\
u(t, x)=0, \quad v(t, x)=0, \quad x \in \partial \Omega, t>0, \\
u(0, x)=u_{0}(x) \geq 0, \quad v(0, x)=v_{0}(x) \geq 0, \quad x \in \Omega .
\end{array}\right.
$$

As we know, the traditional partial differential equations (PDEs) are relations between the values of an unknown function and its derivatives of different orders. If we want to check whether a PDE holds at a particular point, we need to known only the values of the function in an arbitrarily small neighborhood, so that all derivatives can be computed. But a nonlocal equation is a relation for which the opposite happens. In order to check whether a nonlocal equation holds at a point, information as regards the values of the function far from that point is needed. Most of the times, this is because the equation involves integral operators. Usually we call the equations in (1.1) integral-differential equations.

To a great extent, the study of nonlocal equations is motivated by real world applications. In recent years, many authors have investigated the blow-up phenomenon of reactiondiffusion equations with nonlocal source terms or localized source terms subject to Neumann boundary conditions. Wang and Wang [17] considered the nonlocal equation

$$
u_{t}-\Delta u=\int_{\Omega} f(u(t, x)) d x, \quad x \in \Omega, t>0,
$$

and Chadam et al. [18] considered the equation with a localized source term

$$
u_{t}-\Delta u=f\left(u\left(t, x_{0}\right)\right), \quad x_{0}, x \in \Omega, t>0,
$$

with homogeneous Neumann boundary values and nonnegative initial data. Under appropriate conditions, they proved that the solution blows up in finite time and the blow-up is set in $\bar{\Omega}$, respectively.

For the semilinear parabolic system with nonlocal source terms

$$
\begin{cases}u_{t}-\Delta u=\int_{\Omega} \lambda_{1} e^{p_{1} u+q_{1} v} d x, & x \in \Omega, t>0, \\ v_{t}-\Delta v=\int_{\Omega} \lambda_{2} e^{p_{2} u+q_{2} v} d x, & x \in \Omega, t>0,\end{cases}
$$


or with localized source terms

$$
\begin{cases}u_{t}-\Delta u=\lambda_{1} e^{p_{1} u\left(t, x_{0}\right)+q_{1} v\left(t, x_{0}\right)} d x, & x \in \Omega, t>0 \\ v_{t}-\Delta v=\lambda_{2} e^{p_{2} u\left(t, x_{0}\right)+q_{2} v\left(t, x_{0}\right)} d x, & x_{0}, x \in \Omega, t>0\end{cases}
$$

subject to Neumann boundary conditions and initial data, respectively,

$$
\begin{aligned}
& \frac{\partial u}{\partial v}(t, x)=\frac{\partial v}{\partial v}(t, x)=0, \quad x \in \partial \Omega, t>0, \\
& u(0, x)=u_{0}(x), \quad v(0, x)=v_{0}(x), \quad x \in \Omega,
\end{aligned}
$$

where $\Omega \subset R^{N}$ is a bounded domain with smooth boundary $\partial \Omega$, and $v$ is the outward normal on $\partial \Omega$, parameters $\lambda_{i}>0, p_{i}, q_{i} \geq 0(i=1,2), p_{2} q_{1}>0$, the initial functions $u_{0}(x), v_{0}(x) \in C^{1}(\bar{\Omega})$ are nonnegative and satisfy the compatibility conditions $\frac{\partial u_{0}}{\partial v}=\frac{\partial v_{0}}{\partial v}=0$ on $\partial \Omega$.

If $p_{1}=q_{2}=0$, the nonlinear reaction-diffusion systems (1.7) and (1.8) with homogeneous Dirichlet boundary conditions have been studied in Li et al. [19] and Pedersen and Lin [20]. They obtained the blow-up condition and gave the blow-up set $\bar{\Omega}$ and established the asymptotic behavior as

$$
\lim _{t \rightarrow T}|\log (T-t)|^{-1} u(t, x)=1, \quad \lim _{t \rightarrow T}|\log (T-t)|^{-1} v(t, x)=1,
$$

uniformly on $\bar{\Omega}$.

Chen et al. [21] considered the reaction-diffusion systems (1.7) and (1.8) with homogeneous Neumann boundary conditions (1.9) and the initial conditions (1.10). They showed that the solution blows up in finite time $T$, established the estimates of the blow-up rates, and described the asymptotic behavior of the solution.

For the blow-up phenomenon of reaction-diffusion equation on an unbounded domain, there is a classical model:

$$
\begin{cases}u_{t}=\Delta u+u^{1+\alpha}, \quad x \in R^{N}, t>0 \\ u(0, x)=u_{0}(x), \quad x \in R^{N}\end{cases}
$$

On the properties of solution to (1.12) the milestone was the fundamental work of Fujita [22], who proved that (1.12) has no global positive nontrivial if $0<\alpha<\frac{2}{N}$, every solution with arbitrarily small initial data blows up. The same is true for the critical exponent $\alpha=\frac{2}{N}$, as proved by Hayaka [23]. For $\alpha>\frac{2}{N}$, solutions with small initial conditions tend to zero as time $t \rightarrow+\infty$. For $0<1+\alpha \leq 1$ all solutions with bounded initial data are global.

For the generalized quasi-linear equations of the following types:

$$
\begin{aligned}
& u_{t}=\Delta \phi(u)+f(u), \\
& u_{t}=\Delta \phi(u)+f(u,|\nabla u|),
\end{aligned}
$$

the blow-up results can be found in [24] and [25], respectively, for various choices of the functions $\phi$ and $f$.

The nonlocality in the equation can have different sources. In the previous discussion of the nonlocal reaction-diffusion equations, they just contain the nonlocal source terms 
or the nonlocal boundary conditions. Likewise, the most common approach is perhaps to study nonlocal diffusions, often given by a term in the equation which is a linear integrodifferential operator. The equations involving the fractional Laplacian are the prime examples of nonlocal elliptic equations $(-\triangle)^{\frac{\beta}{2}} u=f$ with $\beta \in(0,2)$, where

$$
-(-\triangle)^{\frac{\beta}{2}} u=C_{N, \frac{\beta}{2}} \int_{R^{N}} \frac{u(x+y)-u(x)}{|y|^{N+\beta}} d y, \quad \forall u \in C_{c}^{+\infty}\left(R^{N}\right)
$$

and the integral has to be intended in the principal value sense, $-(-\triangle)^{\frac{\beta}{2}}$ is for integrodifferential equations where $\triangle$ is for elliptic PDEs.

Note that the classical PDEs can be recovered from integro-differential equations in several ways. For example:

$$
\triangle u(x)=\lim _{\beta \rightarrow 2}\left[-(-\triangle)^{\frac{\beta}{2}} u(x)\right]=\lim _{r \rightarrow 0} \frac{C}{r^{N+2}} \int_{B_{r}}[u(x+y)-u(x)] d y .
$$

In this paper we study the Cauchy problem of a fractional reaction-diffusion system (1.1). The blow-up property of solutions to (1.1) was studied much less. Guedda and Kirane [26] considered the blow-up positive solutions to the Cauchy problem of a scalar equation

$$
\left\{\begin{array}{l}
u_{t}+(-\Delta)^{\frac{\beta}{2}} u=h(t) u^{1+\alpha}, \quad x \in R^{N}, t>0 \\
u(0, x)=u_{0}(x) \geq 0, \quad x \in R^{N}
\end{array}\right.
$$

where the nonnegative function $h \in C[0,+\infty)$ satisfies $c_{0} t^{\sigma} \leq h(t) \leq c_{1} t^{\sigma}$ for sufficiently large $t$ and constants $c_{0}, c_{1}>0, \sigma>-1$. Sugitani [27] considered (1.17) for a general nonlinear term $F(u)$ to instead of $u^{1+\alpha}$. When $h \equiv 1$ and $\beta=2$ the problem (1.17) goes back to the problem (1.12), the fundamental work of Fujita [22].

When $\beta=2$ the problem (1.1) goes back to the problem (1.4), the fundamental work was given by Escobedo and Herrero [15, 16]; Uda [28] gave a sufficient condition for blowup of all positive solutions. There are some difficulties in the study of the problem (1.1). First, the fundamental solution $P(t, x)$ of the fractional heat operator $\partial_{t}+(-\triangle)^{\frac{\beta}{2}}$ is more complicated than the fundamental solution $T(t, x)$ of the heat operator $\partial_{t}-\Delta$, this will bring about some obstacles since the fractional power Laplacian is a nonlocal operator. Second, due to the couple of nonlinear source terms $u^{p}(t, x)$ and $v^{q}(t, x)$, it is more difficult to establish the comparison inequalities to get the blow-up condition. Recently, Pérez [29], Pérez and Villa [30, 31], Villa [32] gave a series of results on the blow-up properties of the solutions to the Cauchy problem

$$
\left\{\begin{array}{l}
u_{t}+g_{1}(t)(-\Delta)^{\frac{\alpha}{2}} u=h_{1}(t) v^{p}, \quad x \in R^{N}, t>0, \\
v_{t}+g_{2}(t)(-\Delta)^{\frac{\beta}{2}} v=h_{2}(t) u^{q}, \quad x \in R^{N}, t>0, \\
u(0, x)=u_{0}(x) \geq 0, \quad v(0, x)=v_{0}(x) \geq 0, \quad x \in R^{N} .
\end{array}\right.
$$

Their method was based on the study of blow-up of a particular system of ordinary differential equations; these ordinary differential equations were complicated but were useful to get the blow-up condition.

The treatment in this paper is essentially self-contained and elementary. Different from the method of Pérez and Villa, we use another method to study the blow-up property of 
positive mild solutions to the Cauchy problem (1.1). Our method is based on the fundamental solution $P(t, x)$ of the fractional heat operator $\partial_{t}+(-\triangle)^{\frac{\beta}{2}}$ defined on the whole space $R^{N}$ and is more in the spirit of the properties of $P(t, x)$ established by Yosida [2] and some estimates of $P(t, x)$ developed by Caffarelli and Figalli [5]. We first use an iteration method to establish the estimates of lower bounds of positive mild solutions, then we obtain the unboundedness of solutions for large time. Finally we give a sufficient condition for the positive mild solution to a fractional reaction-diffusion system to blow up in finite time.

The associated integral system of (1.1) is

$$
\left\{\begin{array}{l}
u(t, x)=\int_{R^{N}} P(t, x-y) u_{0}(y) d y+\int_{0}^{t} \int_{R^{N}} P(t-s, x-y) v^{p}(s, y) d y d s \\
v(t, x)=\int_{R^{N}} P(t, x-y) v_{0}(y) d y+\int_{0}^{t} \int_{R^{N}} P(t-s, x-y) u^{q}(s, y) d y d s
\end{array}\right.
$$

We say that $(u, v)$ is a mild solution of $(1.1)$ if $(u, v)$ is a solution of (1.19). In this paper solutions will be understood in the mild sense. The existence of local solutions for the system (1.19) follows from the Banach fixed point theorem. The proof is standard, so we omit it.

The paper is organized as follows. In Section 2, we establish notation and discuss some preliminary material on the fundamental solution $P(t, x)$ of the fractional heat operator $\partial_{t}+(-\triangle)^{\frac{\beta}{2}}$. In Section 3, we prove the blow-up property of the positive mild solution to the problem (1.1). In Section 4, we give a discussion of the study of systems of fractional reaction-diffusion equations.

\section{Preliminaries}

Here we discuss some elementary properties of the fundamental solution $P(t, x)$ of the fractional heat operator $\partial_{t}+(-\triangle)^{\frac{\beta}{2}}$.

Lemma 2.1 [2] Let $P(t, x)$ be the fundamental solution of the fractional heat operator $\partial_{t}+$ $(-\triangle)^{\frac{\beta}{2}}$ in $x \in R^{N}$. Then

$$
\begin{aligned}
& P(t, x)=\int_{0}^{+\infty} f_{t, \frac{\beta}{2}}(s) T(s, x) d s, \quad x \in R^{N}, t>0,0<\beta<2, \\
& P(t, x)=T(t, x)=(4 \pi t)^{-\frac{N}{2}} e^{-\frac{|x|^{2}}{4 t}}, \quad x \in R^{N}, t>0, \beta=2,
\end{aligned}
$$

where $T(t, x)$ is the fundamental solution of the heat operator $\partial_{t}-\triangle$ in $x \in R^{N}$ and

$$
f_{t, \frac{\beta}{2}}(s)=\frac{1}{2 \pi i} \int_{\tau-i \infty}^{\tau+i \infty} e^{z s-t z^{\frac{\beta}{2}}} d z \geq 0, \quad s>0 .
$$

Lemma 2.2 [26] Let $P(t, x)$ be the fundamental solution of the fractional heat operator $\partial_{t}+(-\triangle)^{\frac{\beta}{2}}$ in $x \in R^{N}$. Then we have the following properties:

(a) $P(t s, x)=t^{-\frac{N}{\beta}} P\left(s, t^{-\frac{1}{\beta}} x\right)$,

(b) $P(t, x) \geq\left(\frac{s}{t}\right)^{\frac{N}{\beta}} P(s, x), \forall t \geq s$,

(c) if $P(t, 0) \leq 1$ and $\tau \geq 2$, then $P\left(t, \frac{x-y}{\tau}\right) \geq P(t, x) P(t, y)$,

(d) $\|P(t, \cdot)\|_{L^{1}}=1, \forall t>0$,

and $P(t, 0)$ is decreasing in $t$ and $P(t, x)$ is decreasing in $|x|$. 
Lemma 2.3 [2] The function $f_{t, \frac{\beta}{2}}(s)$ has the following properties.

(1) $\int_{0}^{+\infty} e^{-\lambda a} f_{t, \frac{\beta}{2}}(\lambda) d \lambda=e^{-t a^{\frac{\beta}{2}}}, t>0, a>0$,

(2) $\int_{0}^{+\infty} f_{t, \frac{\beta}{2}}(\lambda) d \lambda=1$,

(3) $f_{t+s, \frac{\beta}{2}}(\lambda)=\int_{0}^{+\infty} f_{t, \frac{\beta}{2}}(\lambda-\mu) f_{s, \frac{\beta}{2}}(\mu) d \mu$,

(4) $\int_{0}^{+\infty} \frac{\partial}{\partial t} f_{t, \frac{\beta}{2}}(\lambda) d \lambda=0, \forall t>0$.

Lemma 2.4 [5] The function $P(t, x)$ is $C^{\infty}$ in $x$ for $t>0$ and there exists a positive constant $C$ such that $P(1, x)$ behaves like $\frac{1}{1+|x|^{N+\beta}}$, which by scaling implies

(1) $C^{-1}\left(t^{-\frac{N}{\beta}} \wedge \frac{t}{|x|^{N+\beta}}\right) \leq P(t, x) \leq C\left(t^{-\frac{N}{\beta}} \wedge \frac{t}{|x|^{N+\beta}}\right)$,

(2) $P(t, x) \approx \frac{t}{t^{\frac{N+\beta}{\beta}}+|x|^{N+\beta}}$.

Lemma 2.5 Let $T(t, x)$ be the fundamental solution of the heat operator $\partial_{t}-\triangle$ in $x \in R^{N}$. Then we have the formula

$$
\int_{R^{N}} T(\tau, x-y) T(\mu, y) d y=T(\tau+\mu, x), \quad \forall \tau>0, \mu>0, x \in R^{N} .
$$

Proof From (2.2), we have

$$
\begin{aligned}
T(\tau, x-y) T(\mu, y) \\
\quad=(4 \pi \tau)^{-\frac{N}{2}}(4 \pi \mu)^{-\frac{N}{2}} e^{-\frac{|x-y|^{2}}{4 \tau}-\frac{|y|^{2}}{4 \mu}} \\
\quad=(4 \pi \tau)^{-\frac{N}{2}}(4 \pi \mu)^{-\frac{N}{2}} e^{-\frac{\tau+\mu}{4 \tau \mu}\left|y-\frac{\mu x}{\tau+\mu}\right|^{2}-\frac{|x|^{2}}{4(\tau+\mu)}},
\end{aligned}
$$

then we have

$$
\begin{aligned}
& \int_{R^{N}} T(\tau, x-y) T(\mu, y) d y \\
& =(4 \pi \tau)^{-\frac{N}{2}}(4 \pi \mu)^{-\frac{N}{2}} e^{-\frac{|x|^{2}}{4(\tau+\mu)}} \int_{R^{N}} e^{-\frac{\tau+\mu}{4 \tau \mu}\left|y-\frac{\mu x}{\tau+\mu}\right|^{2}} d y \\
& =(4 \pi \tau)^{-\frac{N}{2}}(4 \pi \mu)^{-\frac{N}{2}} e^{-\frac{|x|^{2}}{4(\tau+\mu)}} \int_{R^{N}} e^{-\frac{\tau+\mu}{4 \tau \mu}|z|^{2}} d z \\
& =(4 \pi \tau)^{-\frac{N}{2}}(4 \pi \mu)^{-\frac{N}{2}} e^{-\frac{|x|^{2}}{4(\tau+\mu)}}\left(4 \pi \frac{\tau \mu}{\tau+\mu}\right)^{\frac{N}{2}} \\
& =(4 \pi)^{-\frac{N}{2}}(\tau+\mu)^{-\frac{N}{2}} e^{-\frac{|x|^{2}}{4(\tau+\mu)}} \\
& =T(\tau+\mu, x) .
\end{aligned}
$$

The proof is complete.

The following important property is an immediate consequence of the above result.

Lemma 2.6 Let $P(t, x)$ be the fundamental solution of the fractional heat operator $\partial_{t}+$ $(-\triangle)^{\frac{\beta}{2}}$ in $x \in R^{N}$. Then we have

$$
\int_{R^{N}} P(t-s, x-y) P(s, y) d y=P(t, x), \quad \forall t>0, s>0, x \in R^{N} .
$$


Proof From (2.1) and (2.3), we have

$$
\begin{aligned}
\int_{R^{N}} P(t-s, x-y) P(s, y) d y \\
\quad=\int_{R^{N}} \int_{0}^{+\infty} f_{t-s, \frac{\beta}{2}}(\tau) T(\tau, x-y) d \tau \int_{0}^{+\infty} f_{s, \frac{\beta}{2}}(\mu) T(\mu, y) d \mu d y \\
=\int_{0}^{+\infty} d \tau \int_{0}^{+\infty} d \mu \int_{R^{N}} f_{t-s, \frac{\beta}{2}}(\tau) T(\tau, x-y) f_{s, \frac{\beta}{2}}(\mu) T(\mu, y) d y .
\end{aligned}
$$

Lemma 2.5 implies that

$$
\int_{R^{N}} P(t-s, x-y) P(s, y) d y=\int_{0}^{+\infty} \int_{0}^{+\infty} f_{t-s, \frac{\beta}{2}}(\tau) f_{s, \frac{\beta}{2}}(\mu) T(\mu+\tau, x) d \tau d \mu .
$$

Make a transformation in the region $[0,+\infty) \times[0,+\infty): m=\tau, n=\tau+\mu$, the determinant of the Jacobian is $\left|\frac{\partial(m, n)}{\partial(\tau, \mu)}\right|=1$; then we have

$$
\begin{aligned}
\int_{R^{N}} P(t-s, x-y) P(s, y) d y & =\int_{0}^{+\infty} d n \int_{0}^{+\infty} f_{t-s, \frac{\beta}{2}}(m) f_{s, \frac{\beta}{2}}(n-m) T(n, x) d m \\
& =\int_{0}^{+\infty}\left(\int_{0}^{+\infty} f_{t-s, \frac{\beta}{2}}(m) f_{s, \frac{\beta}{2}}(n-m) d m\right) T(n, x) d n
\end{aligned}
$$

using the third property in Lemma 2.3, we have

$$
\int_{R^{N}} P(t-s, x-y) P(s, y) d y=\int_{0}^{+\infty} f_{t, \frac{\beta}{2}}(n) T(n, x) d n=P(t, x) .
$$

The proof is complete.

\section{Blow-up property of positive mild solutions}

In this section, we will study the blow-up property of positive mild solutions to the Cauchy problem (1.1). Let $(u(t, x), v(t, x))$ be a positive mild solution of (1.1). We first prove that the functions

$$
\bar{u}(t)=\int_{R^{N}} P(t, x) u(t, x) d x, \quad \bar{v}(t)=\int_{R^{N}} P(t, x) v(t, x) d x
$$

blow up in a finite time, then we find that the solution $(u(t, x), v(t, x))$ blows up in a finite time.

We first need some preliminary results.

Lemma 3.1 Let $(u(t, x), v(t, x))$ be a positive mild solution of $(1.1)$ and $p, q>1$. Then $(u(t, x), v(t, x))$ blows up in a finite time if and only if $(\bar{u}(t), \bar{v}(t))$ blows up in a finite time, that is, there is a constant $T^{*}>0$ such that $\bar{u}(t)=+\infty, \bar{v}(t)=+\infty$ for all $t \geq T^{*}$.

Remark 3.1 Lemma 3.1 tells us that for $(\bar{u}(t), \bar{v}(t))$ to blow up in a finite time $T^{*}$ implies that $(u(t, x), v(t, x))$ blows up in a finite time $T_{*}>0$; in general, we have $T_{*} \leq T^{*}$. We refer the readers to the paper of Ball [33]. 
Proof Necessity is obvious. We just prove the sufficiency.

Since $\bar{u}(t)=+\infty, \bar{v}(t)=+\infty$ for all $t \geq T^{*}$, we assume that $P\left(T^{*}, 0\right) \leq 1$. The monotonicity of $P(t, 0)$ in Lemma 2.2 implies that $P(t, 0) \leq P\left(T^{*}, 0\right) \leq 1, \forall t \geq T^{*}$.

Let $T^{*} \leq t \leq s \leq \frac{6 t}{2^{\beta}+1}$ and $\tau=\left(\frac{6 t-s}{s}\right)^{\frac{1}{\beta}}$. Following the proof of Lemma 3.1 in [26] we have

$$
P(6 t-s, x-y) \geq\left(\frac{s}{6 t-s}\right)^{\frac{N}{\beta}} P(s, x) P(s, y),
$$

then

$$
\begin{aligned}
& \int_{R^{N}} P(6 t-s, x-y) v(s, y) d y \\
& \quad \geq\left(\frac{s}{6 t-s}\right)^{\frac{N}{\beta}} P(s, x) \int_{R^{N}} P(s, y) v(s, y) d y \\
& =\left(\frac{s}{6 t-s}\right)^{\frac{N}{\beta}} P(s, x) \bar{v}(s),
\end{aligned}
$$

since $T^{*} \leq t \leq s \leq \frac{6 t}{2^{\beta}+1}$ and $\bar{v}(s)=+\infty$, we have

$$
\int_{R^{N}} P(6 t-s, x-y) v(s, y) d y=+\infty .
$$

On the other hand, by the Duhamel formula from the first equation in (1.1), we have

$$
\begin{aligned}
u(6 t, x)= & \int_{R^{N}} P(6 t, x-y) u_{0}(y) d y \\
& +\int_{0}^{6 t} \int_{R^{N}} P(6 t-s, x-y) v^{p}(s, y) d y d s
\end{aligned}
$$

For $p>1$, and $u_{0}(y) \geq 0, v(s, y) \geq 0$, we apply Jensen's inequality to obtain

$$
\begin{aligned}
u(6 t, x) & \geq \int_{0}^{6 t} \int_{R^{N}} P(6 t-s, x-y) v^{p}(s, y) d y \\
& \geq \int_{0}^{\frac{6 t}{2^{\beta}+1}}\left(\int_{R^{N}} P(6 t-s, x-y) v(s, y) d y\right)^{p} d s \\
& =+\infty
\end{aligned}
$$

the last equality comes from (3.2), so that $u(t, x)=+\infty$ for all $t \geq 6 T^{*}$ and $x \in R^{N}$. Similarly, we can prove that $v(t, x)=+\infty$ for all $t \geq 6 T^{*}$ and $x \in R^{N}$. The proof is complete.

Following the proof of Lemma 3.2 in [26], we can get the following result for the coupled system (1.1), a similar result to the case of a scalar equation.

Lemma 3.2 Let $(u(t, x), v(t, x))$ be a positive mild solution of $(1.1)$. There exist positive constants $t_{0}, c$ such that for $\delta=\frac{t_{0}}{2^{\beta}}$

$$
\begin{cases}u\left(t_{0}, x\right) \geq c P(\delta, x), & x \in R^{N} \\ v\left(t_{0}, x\right) \geq c P(\delta, x), & x \in R^{N}\end{cases}
$$


Lemma 3.3 Let $(u(t, x), v(t, x))$ be a positive mild solution of $(1.1), t_{0}, c_{0}$ be positive constants and $l_{1}, l_{2}$ be constants. Assume that for all $x \in R^{N}, t \geq t_{0}$

$$
\begin{cases}u(t, x) \geq c_{0} t^{l_{1}} P(t, x), & x \in R^{N}, \\ v(t, x) \geq c_{0} t^{l_{2}} P(t, x), & x \in R^{N}\end{cases}
$$

(1) There exist positive constants $t_{1} \geq 2 t_{0}, c_{1}$ such that for $x \in R^{N}, t \geq t_{1}$

$$
\begin{cases}u(t, x) \geq c_{1} t^{1+l_{2} p} P^{p}(t, x), & 1+l_{2} p \neq 0 \\ v(t, x) \geq c_{1} t^{1+l_{1} q} P^{q}(t, x), & 1+l_{1} q \neq 0\end{cases}
$$

(2) There exist positive constants $t_{1} \geq t_{0}^{2}, c_{1}$ such that for $x \in R^{N}, t \geq t_{1}$

$$
\begin{cases}u(t, x) \geq c_{1} \ln t P^{p}(t, x), & 1+l_{2} p=0 \\ v(t, x) \geq c_{1} \ln t P^{q}(t, x), & 1+l_{1} q=0 .\end{cases}
$$

Proof By the Duhamel formula of the second equation in (1.1), we have

$$
\begin{aligned}
v(t, x)= & \int_{R^{N}} P\left(t-t_{0}, x-y\right) u\left(t_{0}, y\right) d y \\
& +\int_{t_{0}}^{t} \int_{R^{N}} P(t-s, x-y) u^{q}(s, y) d y d s \\
\geq & \int_{t_{0}}^{t} \int_{R^{N}} P(t-s, x-y) u^{q}(s, y) d y d s .
\end{aligned}
$$

Thanks to Jensen's inequality, (3.4) implies that

$$
\begin{aligned}
v(t, x) & \geq \int_{t_{0}}^{t}\left(\int_{R^{N}} P(t-s, x-y) u(s, y) d y\right)^{q} d s \\
& \geq c_{0}^{q} \int_{t_{0}}^{t} s^{l_{1} q}\left(\int_{R^{N}} P(t-s, x-y) P(s, y) d y\right)^{q} d s,
\end{aligned}
$$

by the property of $P(t, x)$ in Lemma 2.6, (2.5) implies that

$$
\begin{aligned}
v(t, x) & \geq c_{0}^{q} \int_{t_{0}}^{t} s^{l_{1} q} P^{q}(t, x) d s \\
& =c_{0}^{q} P^{q}(t, x) \int_{t_{0}}^{t} s^{l_{1} q} d s \\
& =\frac{c_{0}^{q}}{1+l_{1} q} P^{q}(t, x)\left[t^{1+l_{1} q}-t_{0}^{1+l_{1} q}\right] .
\end{aligned}
$$

Three cases arise.

Case 1. If $1+l_{1} q>0$, there exists a constant $t_{1} \geq 2 t_{0}$ such that for all $t \geq t_{1}$ we have $t_{0} \leq \frac{t}{2}$ and

$$
t_{0}^{1+l_{1} q} \leq\left(\frac{t}{2}\right)^{1+l_{1} q}=\frac{t^{1+l_{1} q}}{2^{1+l_{1} q}}
$$


then we have

$$
v(t, x) \geq \frac{c_{0}^{q}}{1+l_{1} q}\left(1-\frac{1}{2^{1+l_{1} q}}\right) t^{1+l_{1} q} P^{q}(t, x) \quad \text { for all } x \in R^{N}, t \geq t_{1} .
$$

Denote the constant $c_{1}=\frac{c_{0}^{q}}{1+l_{1} q}\left(1-\frac{1}{2^{1+l_{1} q}}\right)>0$, we get

$$
v(t, x) \geq c_{1} t^{1+l_{1} q} P^{q}(t, x) \quad \text { for all } x \in R^{N}, t \geq t_{1} .
$$

Case 2. If $1+l_{1} q<0$, there exists a constant $t_{1} \geq 2 t_{0}$ such that for all $t \geq t_{1}$ we have $t_{0} \leq \frac{t}{2}$ and

$$
v(t, x) \geq \frac{c_{0}^{q}}{-\left(1+l_{1} q\right)}\left(2^{-\left(1+l_{1} q\right)}-1\right) t^{1+l_{1} q} P^{q}(t, x) \quad \text { for all } x \in R^{N}, t \geq t_{1} .
$$

Denote the constant $c_{1}=\frac{c_{0}^{q}}{-\left(1+l_{1} q\right)}\left(2^{-\left(1+l_{1} q\right)}-1\right)>0$, we get

$$
v(t, x) \geq c_{1} t^{1+l_{1} q} P^{q}(t, x) \quad \text { for all } x \in R^{N}, t \geq t_{1} .
$$

Case 3. If $1+l_{1} q=0$, there exists a constant $t_{1} \geq t_{0}^{2}$ such that for all $t \geq t_{1}$ we have $t_{0} \leq \sqrt{t}$, that is, $\ln t_{0} \leq \frac{1}{2} \ln t$, then

$$
\begin{aligned}
v(t, x) & \geq c_{0}^{q} P^{q}(t, x)\left(\ln t-\ln t_{0}\right) \\
& \geq \frac{c_{0}^{q}}{2} P^{q}(t, x) \ln t \quad \text { for all } x \in R^{N}, t \geq t_{1} .
\end{aligned}
$$

Denote the constant $c_{1}=\frac{c_{0}^{q}}{2}>0$, we get

$$
v(t, x) \geq c_{1} \ln t P^{q}(t, x) \quad \text { for all } x \in R^{N}, t \geq t_{1} .
$$

According to the above three cases, we get for all $x \in R^{N}, t \geq t_{1}$

$$
\left\{\begin{array}{l}
v(t, x) \geq c_{1} t^{1+l_{1} q} P^{q}(t, x), \quad 1+l_{1} q \neq 0 \\
u(t, x) \geq c_{1} \ln t P^{q}(t, x), \quad 1+l_{1} q=0
\end{array}\right.
$$

Similarly, we can prove that for $x \in R^{N}, t \geq t_{1}$

$$
\left\{\begin{array}{l}
u(t, x) \geq c_{1} t^{1+l_{2} p} P^{p}(t, x), \quad 1+l_{2} p \neq 0 \\
u(t, x) \geq c_{1} \ln t P^{p}(t, x), \quad 1+l_{2} p=0
\end{array}\right.
$$

This concludes the proof.

Theorem 3.1 Let $(u(t, x), v(t, x))$ be a positive mild solution of (1.1). Assume $p>1, q>1$, and $p q<1+\frac{\beta}{2 N}(1+\min \{p, q\})$, then

$$
\lim _{t \rightarrow+\infty} u(t, x)=+\infty, \quad \lim _{t \rightarrow+\infty} v(t, x)=+\infty
$$


Proof Without loss of generality, we assume that $u_{0}(x) \not \equiv 0$ and $v_{0}(x) \not \equiv 0$. Otherwise, we can translate the time axis and choose a suitable time as the initial time. On the other hand, we will prove the result only for $1+l_{1} q \neq 0$ and $1+l_{2} p \neq 0$. For $1+l_{1} q=0$ or $1+l_{2} p=0$, the proof is very similar.

By Lemma 3.2 and the Duhamel formula we get

$$
\begin{aligned}
u\left(t+t_{0}, x\right) & \geq \int_{R^{N}} P(t, x-y) u\left(t_{0}, y\right) d y \\
& \geq c \int_{R^{N}} P(t, x-y) P(\delta, y) d y \\
& =c P(t+\delta, x), \quad \forall x \in R^{N}, t \geq 0,
\end{aligned}
$$

then

$$
u(t, x) \geq c P\left(t-t_{0}+\delta, x\right), \quad \forall x \in R^{N}, t \geq t_{0},
$$

due to the property in Lemma $2.2, \forall x \in R^{N}, t \geq t_{0}$,

$$
\begin{aligned}
u(t, x) & \geq c\left(\frac{t}{t-t_{0}+\delta}\right)^{\frac{N}{\beta}} P(t, x) \\
& \geq c t_{0}^{\frac{N}{\beta}} t^{-\frac{N}{\beta}} P(t, x) \\
& =c t^{-\frac{N}{\beta}} P(t, x),
\end{aligned}
$$

that is,

$$
u(t, x) \geq c t^{-\frac{N}{\beta}} P(t, x), \quad \forall x \in R^{N}, t \geq t_{0} .
$$

Similarly, we have

$$
v(t, x) \geq c t^{-\frac{N}{\beta}} P(t, x), \quad \forall x \in R^{N}, t \geq t_{0} .
$$

From (3.8), (3.9), and Lemma 3.3, there exist constants $c_{1}, t_{1}$ such that

$$
\begin{aligned}
& u(t, x) \geq c_{1} t^{1-\frac{N}{\beta} p} P^{p}(t, x), \quad \forall x \in R^{N}, t \geq t_{1}, \\
& v(t, x) \geq c_{1} t^{1-\frac{N}{\beta} q} P^{q}(t, x), \quad \forall x \in R^{N}, t \geq t_{1} .
\end{aligned}
$$

We claim that for any positive integer $k$ there exist constants $c_{k}, t_{k}$ such that for an even number $k$

$$
\begin{array}{ll}
u(t, x) \geq c_{k} t^{(1+p) \frac{(p q)^{\frac{k}{2}}-1}{p q-1}-\frac{N}{\beta}(p q)^{\frac{k}{2}}} P^{(p q)^{\frac{k}{2}}}(t, x), & \forall x \in R^{N}, t \geq t_{k}, \\
v(t, x) \geq c_{k} t^{(1+q) \frac{(p q) \frac{k}{2}-1}{p q-1}-\frac{N}{\beta}(p q)^{\frac{k}{2}}} P^{(p q)^{\frac{k}{2}}}(t, x), & \forall x \in R^{N}, t \geq t_{k},
\end{array}
$$


and for an odd number $k$ :

$$
\begin{array}{ll}
u(t, x) \geq c_{k} t^{(1+p) \frac{(p q))^{\frac{k-1}{2}}-1}{p q-1}+(p q)^{\frac{k-1}{2}}\left(1-\frac{N}{\beta} p\right)} P^{(p q)^{\frac{k-1}{2}} p}(t, x), & \forall x \in R^{N}, t \geq t_{k}, \\
v(t, x) \geq c_{k} t^{(1+q) \frac{(p q))^{\frac{k-1}{2}}-1}{p q-1}+(p q)^{\frac{k-1}{2}}\left(1-\frac{N}{\beta} q\right)} P^{(p q)^{\frac{k-1}{2}}}(t, x), & \forall x \in R^{N}, t \geq t_{k} .
\end{array}
$$

Equations (3.10) and (3.11) imply that (3.12) and (3.13) hold for $k=1$. We assume that (3.12) and (3.13) hold for $k=2 r-1$ :

$$
\begin{aligned}
& u(t, x) \geq c_{2 r-1} t^{(1+p) \frac{(p q)^{r-1}-1}{p q-1}+(p q)^{r-1}\left(1-\frac{N}{\beta} p\right)} P^{(p q)^{r-1} p}(t, x), \quad \forall x \in R^{N}, t \geq t_{k}, \\
& v(t, x) \geq c_{2 r-1} t^{(1+q) \frac{(p q)^{r-1}-1}{p q-1}+(p q)^{r-1}\left(1-\frac{N}{\beta} q\right)} P^{(p q)^{r-1} q}(t, x), \quad \forall x \in R^{N}, t \geq t_{k} ;
\end{aligned}
$$

then for $k=2 r$, from (3.16), (3.17), and Lemma 3.3, there exist constants $c_{2 r}, t_{2 r}$ such that

$$
\begin{aligned}
u(t, x) & \geq c_{2 r} t^{1+p(1+q) \frac{(p q)^{r-1}-1}{p q-1}+p(p q)^{r-1}\left(1-\frac{N}{\beta} q\right)} P^{(p q)^{r}}(t, x) \\
& \geq c_{2 r} t^{(1+p) \frac{(p q))^{r}-1}{p q-1}-(p q)^{r} \frac{N}{\beta}} P^{(p q)^{r}}(t, x) .
\end{aligned}
$$

Similarly we can get

$$
\begin{aligned}
v(t, x) & \geq c_{2 r} t^{1+q(1+p) \frac{(p q)^{r-1}-1}{p q-1}+q(p q)^{r-1}\left(1-\frac{N}{\beta} p\right)} P^{(p q)^{r}}(t, x) \\
& \geq c_{2 r} t^{(1+q) \frac{(p q)^{r}-1}{p q-1}-(p q)^{r} \frac{N}{\beta}} P^{(p q)^{r}}(t, x) .
\end{aligned}
$$

It is easy to check that (3.12) and (3.13) hold for $k=2$. We assume that (3.12) and (3.13) hold for $k=2 r$, similarly we can check that (3.12) and (3.13) hold for $k=2 r+1$.

Now for an even number $k$, using Lemma 2.4 we have

$$
u(t, x) \geq c_{k} t^{(1+p) \frac{(p q)}{p q-1}^{\frac{k}{2}-1}-\frac{N}{\beta}(p q)^{\frac{k}{2}}}\left(\frac{t}{t^{\frac{N+\beta}{\beta}}+|x|^{N+\beta}}\right)^{(p q)^{\frac{k}{2}}} .
$$

Fix $x \in R^{N}$, for large enough $t$, we get

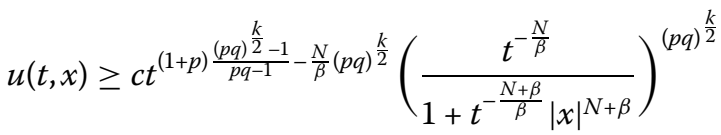

$$
\begin{aligned}
& \geq c t^{(1+p) \frac{(p q)^{\frac{k}{2}}-1}{p q-1}-2 \frac{N}{\beta}(p q)^{\frac{k}{2}}}=c t^{A},
\end{aligned}
$$

where the power of $t$ is

$$
\begin{aligned}
A & =(1+p) \frac{(p q)^{\frac{k}{2}}-1}{p q-1}-\frac{2 N}{\beta}(p q)^{\frac{k}{2}} \\
& =\frac{1}{p q-1}\left\{(p q)^{\frac{k}{2}}\left[1+p-\frac{2 N}{\beta}(p q-1)\right]-(1+p)\right\} .
\end{aligned}
$$


The conditions $p>1, q>1$, and $p q<1+\frac{\beta}{2 N}(1+\min \{p, q\})$ imply that $1+p-\frac{2 N}{\beta}(p q-1)>0$, then $A \rightarrow+\infty$ as $k \rightarrow+\infty$.

Therefore, we have

$$
\lim _{t \rightarrow+\infty} u(x, t)=+\infty
$$

uniformly in $x \in R^{N}$.

Similarly we can get

$$
\lim _{t \rightarrow+\infty} v(x, t)=+\infty
$$

uniformly in $x \in R^{N}$.

Finally, for an odd number $k$, (3.14) implies that

$$
u(t, x) \geq c t^{(1+p) \frac{(p q) \frac{k-1}{2}}{p q-1}+(p q)^{\frac{k-1}{2}}\left(1-\frac{N}{\beta} p\right)}\left(\frac{t^{-\frac{N}{\beta}}}{1+t^{-\frac{N+\beta}{\beta}}|x|^{N+\beta}}\right)^{(p q)^{\frac{k-1}{2}} p} .
$$

Fix $x \in R^{N}$, for large enough $t$, we get

$$
u(t, x) \geq c t^{(1+p) \frac{(p q)^{\frac{k-1}{2}}-1}{p q-1}+(p q)^{\frac{k-1}{2}}\left(1-\frac{2 N}{\beta} p\right)}=c t^{B},
$$

where the exponent is

$$
\begin{aligned}
B & =(1+p) \frac{(p q)^{\frac{k-1}{2}}-1}{p q-1}+(p q)^{\frac{k-1}{2}}\left(1-\frac{2 N}{\beta} p\right) \\
& =\frac{1}{p q-1}\left\{(p q)^{\frac{k-1}{2}} p\left[1+q-\frac{2 N}{\beta}(p q-1)\right]-(1+q)\right\} .
\end{aligned}
$$

The conditions $p>1, q>1$, and $p q<1+\frac{\beta}{2 N}(1+\min \{p, q\})$ imply that $1+q-\frac{2 N}{\beta}(p q-1)>0$, then $B \rightarrow+\infty$ as $k \rightarrow+\infty$.

Therefore, we have

$$
\lim _{t \rightarrow+\infty} u(x, t)=+\infty
$$

uniformly in $x \in R^{N}$ for an odd number $k$.

Similarly we can prove that

$$
\lim _{t \rightarrow+\infty} v(x, t)=+\infty
$$

uniformly in $x \in R^{N}$ for an odd number $k$.

The proof of the theorem is complete.

Theorem 3.2 Let $(u(t, x), v(t, x))$ be a positive mild solution of (1.1). Assume $p>1, q>1$, and $p q<1+\frac{\beta}{2 N}(1+\min \{p, q\})$, then $(u(t, x), v(t, x))$ blows up in a finite time $T_{0}>0 ; u(t, x)=+\infty$, $v(t, x)=+\infty$ for all $t \geq T_{0}$ and $x \in R^{N}$. 
Proof By the Duhamel formula from the first equation in (1.1), we have

$$
\begin{aligned}
u\left(t+t_{0}, x\right)= & \int_{R^{N}} P(t, x-y) u\left(t_{0}, y\right) d y \\
& +\int_{t_{0}}^{t+t_{0}} \int_{R^{N}} P\left(t+t_{0}-s, x-y\right) \nu^{p}(s, y) d y d s,
\end{aligned}
$$

using the definition of $\bar{u}(t)$ in (3.1) we have

$$
\begin{aligned}
\bar{u}\left(t+t_{0}\right)= & \int_{R^{N}} \int_{R^{N}} P\left(t+t_{0}, x\right) P(t, x-y) d x u\left(t_{0}, y\right) d y \\
& +\int_{t_{0}}^{t+t_{0}} \int_{R^{N}} \int_{R^{N}} P\left(t+t_{0}, x\right) P\left(t+t_{0}-s, x-y\right) d x v^{p}(s, y) d y d s,
\end{aligned}
$$

by Lemma 2.6 and Lemma 3.2,

$$
\begin{aligned}
\bar{u}\left(t+t_{0}\right)= & \int_{R^{N}} P\left(2 t+t_{0}, y\right) u\left(t_{0}, y\right) d y \\
& +\int_{t_{0}}^{t+t_{0}} \int_{R^{N}} P\left(2 t+2 t_{0}-s, y\right) v^{p}(s, y) d y d s \\
\geq & c \int_{R^{N}} P\left(2 t+t_{0}, y\right) P(\delta, y) d y \\
& +\int_{0}^{t} \int_{R^{N}} P\left(2 t+t_{0}-s, y\right) v^{p}\left(s+t_{0}, y\right) d y d s .
\end{aligned}
$$

Due to Lemma 2.2, Lemma 3.2, and Jensen's inequality, we get

$$
\begin{aligned}
\bar{u}\left(t+t_{0}\right) \geq & c P(1,0)\left(2 t+t_{0}+\delta\right)^{-\frac{N}{\beta}} \\
& +\int_{0}^{t}\left(\frac{s+t_{0}}{2 t+t_{0}-s}\right)^{\frac{N}{\beta}} \bar{v}^{p}\left(s+t_{0}\right) d s, \quad \forall t \geq 0,
\end{aligned}
$$

then, for $t \geq t_{0}$,

$$
\bar{u}(t) \geq c P(1,0)\left(2 t-t_{0}+\delta\right)^{-\frac{N}{\beta}}+(2 t)^{-\frac{N}{\beta}} \int_{t_{0}}^{t} s^{\frac{N}{\beta}} \bar{v}^{p}(s) d s,
$$

that is,

$$
t^{\frac{N}{\beta}} \bar{u}(t) \geq c P(1,0)\left(\frac{t}{2 t-t_{0}+\delta}\right)^{\frac{N}{\beta}}+2^{-\frac{N}{\beta}} \int_{t_{0}}^{t} s^{\frac{N}{\beta}} \bar{v}^{p}(s) d s,
$$

hence, for $t \geq t_{0}$, we have

$$
t^{\frac{N}{\beta}} \bar{u}(t) \geq c 2^{-\frac{N}{\beta}} P(1,0)+2^{-\frac{N}{\beta}} \int_{t_{0}}^{t} s^{\frac{N}{\beta}} \bar{\nu}^{p}(s) d s .
$$

Similarly for $t \geq t_{0}$, we also have

$$
t^{\frac{N}{\beta}} \bar{v}(t) \geq c 2^{-\frac{N}{\beta}} P(1,0)+2^{-\frac{N}{\beta}} \int_{t_{0}}^{t} s^{\frac{N}{\beta}} \bar{u}^{q}(s) d s .
$$


From $p>1, q>1$, we know that $r=\min \{p, q\}>1$. Theorem 3.1 implies that $u(t, x)>1$, $v(t, x)>1$ for large enough $t$. We add (3.26) and (3.27):

$$
\begin{aligned}
t^{\frac{N}{\beta}} & {[\bar{u}(t)+\bar{v}(t)] } \\
& \geq c 2^{1-\frac{N}{\beta}} P(1,0)+2^{-\frac{N}{\beta}} \int_{t_{0}}^{t} s^{\frac{N}{\beta}}\left[\bar{v}^{p}(s)+\bar{u}^{q}(s)\right] d s \\
& \geq c 2^{1-\frac{N}{\beta}} P(1,0)+2^{-\frac{N}{\beta}} \int_{t_{0}}^{t} s^{\frac{N}{\beta}}\left[\bar{v}^{r}(s)+\bar{u}^{r}(s)\right] d s \\
& \geq c 2^{1-\frac{N}{\beta}} P(1,0)+2^{-\frac{N}{\beta}} \int_{t_{0}}^{t} s^{\frac{N}{\beta}} 2^{1-r}[\bar{v}(s)+\bar{u}(s)]^{r} d s \\
& =c_{1}+c_{2} \int_{t_{0}}^{t} s^{\frac{N(1-r)}{\beta}} 2^{1-r}\left[s^{\frac{N}{\beta}} \bar{v}(s)+s^{\frac{N}{\beta}} \bar{u}(s)\right]^{r} d s,
\end{aligned}
$$

where $c_{1}, c_{2}$ are positive constants.

Set

$$
f(t)=t^{\frac{N}{\beta}}[\bar{u}(t)+\bar{v}(t)], \quad t \geq t_{0}
$$

Equation (3.28) becomes

$$
f(t) \geq c_{1}+c_{2} \int_{t_{0}}^{t} s^{\frac{N(1-r)}{\beta}} 2^{1-r} f^{r}(s) d s .
$$

\section{Denote}

$$
g(t)=c_{1}+c_{2} \int_{t_{0}}^{t} s^{\frac{N(1-r)}{\beta}} 2^{1-r} g^{r}(s) d s .
$$

Then $g(t)$ is a solution of the initial value problem

$$
\left\{\begin{array}{l}
g^{\prime}(t)=c_{2} t^{\frac{N(1-r)}{\beta}} g^{r}(t) \\
g\left(t_{0}\right)=c_{1}
\end{array}\right.
$$

Its solution is

$$
g(t)=\frac{1}{\left[c_{1}^{1-r}+\frac{c_{2}(r-1)}{1-\frac{N}{\beta}(r-1)} t_{0}^{1-\frac{N}{\beta}(r-1)}-\frac{c_{2}(r-1)}{1-\frac{N}{\beta}(r-1)} t^{1-\frac{N}{\beta}(r-1)}\right]^{r-1}} .
$$

Set

$$
T_{0}=\left[\frac{c_{1}^{1-r}\left(1-\frac{N}{\beta}(r-1)\right)}{c_{2}(r-1)}+t_{0}^{1-\frac{N}{\beta}(r-1)}\right]^{\frac{1}{1-\frac{N}{\beta}(r-1)}}
$$

If $q \geq p>1$ and $p q<1+\frac{\beta}{2 N}(1+\min \{p, q\})$, then $r=p>1$ and

$$
r=\frac{p q}{q}<\frac{1}{q}+\frac{\beta}{2 N} \frac{1+\min \{p, q\}}{q}<1+\frac{\beta}{N},
$$

that is, $1-\frac{N}{\beta}(r-1)>0$, then $T_{0}<+\infty$. 


$$
\text { If } \begin{aligned}
p & \geq q>1 \text { and } p q<1+\frac{\beta}{2 N}(1+\min \{p, q\}) \text {, then } r=q>1 \text { and } \\
r & =\frac{p q}{p}<\frac{1}{p}+\frac{\beta}{2 N} \frac{1+\min \{p, q\}}{p}<1+\frac{\beta}{N},
\end{aligned}
$$

that is, $1-\frac{N}{\beta}(r-1)>0$, then $T_{0}<+\infty$.

Therefore, the conditions $p>1, q>1$, and $p q<1+\frac{\beta}{2 N}(1+\min \{p, q\})$ imply that $T_{0}<+\infty$ and

$$
\lim _{t \uparrow T_{0}} g(t)=\lim _{t \uparrow T_{0}}\left[\frac{1-\frac{N}{\beta}(r-1)}{c_{2}(r-1)\left(T_{0}^{1-\frac{N}{\beta}(r-1)}-t^{1-\frac{N}{\beta}(r-1)}\right)}\right]^{r-1}=+\infty
$$

The comparison principle implies that $f(t) \geq g(t)$, so we have

$$
\lim _{t \uparrow T_{0}} f(t)=+\infty
$$

From (3.28), we get

$$
\lim _{t \uparrow T_{0}} \bar{u}(t)=+\infty, \quad \lim _{t \uparrow T_{0}} \bar{v}(t)=+\infty
$$

Therefore, Lemma 3.1 implies that $(u(t, x), v(t, x))$ blows up in a finite time $T_{0}>0 ; u(t, x)=$ $+\infty, v(t, x)=+\infty$ for $t \geq T_{0}$ and $x \in R^{N}$.

This will conclude the proof of Theorem 3.2.

\section{Discussion}

In this paper, we investigated the blow-up property of the positive mild solutions to a system of fractional reaction-diffusion equations,

$$
\left\{\begin{array}{l}
u_{t}+(-\Delta)^{\frac{\beta}{2}} u=v^{p}, \quad x \in R^{N}, t>0, \\
v_{t}+(-\Delta)^{\frac{\beta}{2}} v=u^{q}, \quad x \in R^{N}, t>0, \\
u(0, x)=u_{0}(x) \geq 0, \quad v(0, x)=v_{0}(x) \geq 0, \quad x \in R^{N},
\end{array}\right.
$$

where $p, q$ are positive constants, and the fractional power of the Laplacian operator $(-\Delta)^{\frac{\beta}{2}}$ for $0<\beta \leq 2$ is a nonlocal operator. The asymptotic behavior of solutions to the system (4.1) was studied much less. The main difficulty is the complicated expression of the fundamental solution $P(t, x)$ of the fractional heat operator $\partial_{t}+(-\triangle)^{\frac{\beta}{2}}$. Using the properties of the fundamental solution $P(t, x)$ and some estimates of $P(x, t)$ developed by Yosida [2], Caffarelli and Figalli [5], and Guedda and Kirane [26], we give a sufficient condition that the positive mild solution of the fractional reaction-diffusion system blows up in finite time.

Maybe one can use the method described in this paper to consider the blow-up solutions to the following Cauchy problem of a fractional reaction-diffusion system:

$$
\left\{\begin{array}{l}
u_{t}+g_{1}(t)(-\Delta)^{\frac{\alpha}{2}} u=h_{1}(t)|x|^{m} v^{p}, \quad x \in R^{N}, t>0, \\
v_{t}+g_{2}(t)(-\Delta)^{\frac{\beta}{2}} v=h_{2}(t)|x|^{n} u^{q}, \quad x \in R^{N}, t>0, \\
u(0, x)=u_{0}(x) \geq 0, \quad v(0, x)=v_{0}(x) \geq 0, \quad x \in R^{N},
\end{array}\right.
$$


where $0<\alpha, \beta \leq 2, g_{i}(t), h_{i}(t) \in C(0,+\infty)(i=1,2)$ are nonnegative functions and $h_{i}(t)$ $(i=1,2)$ satisfy

$$
c_{0 i} t^{\sigma_{i}} \leq h_{i}(t) \leq c_{1 i} t^{\sigma_{i}}
$$

for large enough $t, c_{0 i}>0, c_{1 i}>0, \sigma_{i}>-1(i=1,2)$ are constants.

For more details on the model (4.2) one can refer to Pérez [29-31] and Villa [32].

Competing interests

The authors declare that they have no competing interests.

\section{Authors' contributions}

EW carried out the blow-up property of solutions. YT carried out the properties of fractional heat kernel. All authors read and approved the final manuscript.

\section{Acknowledgements}

This work was supported by the National Natural Science Foundation of China grant 11471129.

Received: 22 October 2014 Accepted: 24 March 2015 Published online: 08 April 2015

\section{References}

1. Shlesinger, MF, Zaslavsky, GM, Frisch, U (eds.): Lévy Flights and Related Topics in Physics. Lecture Notes in Physics, vol. 450. Springer, Berlin (1995)

2. Yosida, H: Functional Analysis. Springer, Berlin (1966)

3. Caffarelli, LA, Salsa, S, Silvestre, L: Regularity estimates for the solution and the free boundary of the obstacle problem for the fractional Laplacian. Invent. Math. 171(2), 425-461 (2008)

4. Caffarelli, LA, Vasseur, A: Drift diffusion equations with fractional diffusion and the quasi-geostrophic equation Ann. Math. (2) 171(3), 1903-1930 (2010)

5. Caffarelli, LA, Figalli, A: Regularity of solutions to parabolic fractional obstacle problem. J. Reine Angew. Math. 680 , 191-233 (2013)

6. Wang, $M$, Tang, YB: Long time dynamics of $2 \mathrm{D}$ quasi-geostrophic equations with damping in $L^{p}$. J. Math. Anal. Appl. 412, 866-877 (2014)

7. Wang, $M$, Tang, YB: On dimension of the global attractor for 2D quasi-geostrophic equations. Nonlinear Anal., Real World Appl. 14, 1887-1895 (2013)

8. Chen, T, Chen, Z, Tang, YB: Finite dimensionality of global attractors for a non-classical reaction-diffusion equation with memory. Appl. Math. Lett. 25(3), 357-362 (2012)

9. Tang, YB, Zhou, L: Stability switch and Hopf bifurcation for a diffusive prey predator system with delay. J. Math. Anal. Appl. 334(2), 1290-1307 (2007)

10. Wang, $M$, Tang, YB: Attractors in $H^{2}$ and $L^{2 p-2}$ for reaction diffusion equations on unbounded domains. Commun. Pure Appl. Anal. 12(2), 1111-1121 (2013)

11. Wu, EZ, Tang, YB: Random perturbations of reaction-diffusion waves in biology. Wave Motion 49(7), 632-637 (2012)

12. Zhou, L, Tang, YB, Hussein, S: Stability and Hopf bifurcation for a delay competition diffusion system. Chaos Solitons Fractals 14, 1201-1225 (2002)

13. Guo, YT, Tang, YB: Blow-up for the weakly dissipative generalized Camassa-Holm equation. J. Inequal. Appl. 2014, 514 (2014)

14. Kaplan, S: On the growth of solutions of quasilinear parabolic equations. Commun. Pure Appl. Math. 16, 305-333 (1963)

15. Escobedo, M, Herrero, MA: A semilinear parabolic system in a bounded domain. Ann. Mat. Pura Appl. 165(1), 315-336 (1993)

16. Escobedo, M, Herrero, MA: Boundedness and blow up for a semilinear reaction diffusion system. J. Differ. Equ. 89 176-202 (1991)

17. Wang, MX, Wang, YM: Properties of positive solutions of nonlocal reaction diffusion system. Math. Methods Appl. Sci. 19(14), 1141-1156 (1996)

18. Chadam, JM, Peirce, A, Yin, HM: The blowup property of the solutions to some diffusion equations with localized nonlinear reactions. J. Math. Anal. Appl. 169(2), 313-328 (1992)

19. Li, FC, Chen, YP, Xie, CH: Asymptotic behavior of solution for nonlocal reaction diffusion system. Acta Math. Sci. 23, 261-273 (2003)

20. Pedersen, M, Lin, ZG: The profile near blowup time for solutions of diffusion systems coupled with localized nonlinear reactions. Nonlinear Anal. 50, 1013-1024 (2002)

21. Chen, Q, Hu, XG, Xia, AY: The blowup properties of solutions to a nonlocal reaction diffusion system. J. Sichuan Univ. Natur. Sci. Ed. 43(5), 962-966 (2006)

22. Fujita, $\mathrm{H}$ : On the blowing up of solutions to the Cauchy problem for $u_{t}=\Delta u+u^{1+\alpha}$. J. Fac. Sci., Univ. Tokyo, Sect. $1 \mathrm{~A}$, Math. 13(1), 109-124 (1966)

23. Hayaka, K: On nonexistence of global solutions of some semilinear parabolic equations. Proc. Jpn. Acad. 49, 503-505 (1973)

24. Samarskii, AA: Blowup in Quasilinear Parabolic Equations. de Gruyter, Berlin (1995) 
25. Souplet, P: Recent results and open problems on parabolic equations with gradient nonlinearities. Electron. J. Differ. Equ. 2001, 20 (2001)

26. Guedda, M, Kirane, M: A note on nonexistence of global solutions to a nonlinear integral equation. Bull. Belg. Math. Soc. Simon Stevin 6, 491-497 (1999)

27. Sugitani, S: On nonexistence of global solutions for some nonlinear integral equations. Osaka J. Math. 12, 45-51 (1975)

28. Uda, Y: The critical exponent for a weakly coupled system of the generalized Fujita type reaction-diffusion equations. Z. Angew. Math. Phys. 46, 366-383 (1995)

29. Pérez, A: A blow up condition for a nonautonomous semilinear system. Electron. J. Differ. Equ. 2006, 94 (2006)

30. Pérez, A, Villa, J: A note on blow-up of a nonlinear integral equation. Bull. Belg. Math. Soc. Simon Stevin 17, 891-897 (2010)

31. Pérez, A, Villa, J: Blow-up for a system with time-dependent generators. ALEA Lat. Am. J. Probab. Math. Stat. 7, 207-215 (2010)

32. Villa, J: Blow up of mild solutions of a system of partial differential equations with distinct fractional diffusions. Electron. J. Differ. Equ. 2014, 41 (2014)

33. Ball, JM: Remarks on blowup and nonexistence theorems for nonlinear evolution equations. Q. J. Math. 28(2), 473-486 (1977)

Submit your manuscript to a SpringerOpen ${ }^{\odot}$ journal and benefit from:

- Convenient online submission

- Rigorous peer review

- Immediate publication on acceptance

- Open access: articles freely available online

- High visibility within the field

- Retaining the copyright to your article 